\title{
The Theory and Observational Evidence for Streamlets: A New Velocity-Based Feature Model of Jet Streams and Eddies in the Oceans
}

\author{
Alexander V. Kazansky and Antonina A. Shupikova \\ Far East Division of the Russian Academy of Sciences, Institute of Automation and Control Processes, Radio Street 5, \\ Vladivostok 690041, Russia \\ Correspondence should be addressed to Alexander V. Kazansky; kazansky@iacp.dvo.ru
}

Received 17 August 2012; Accepted 7 September 2012

Academic Editors: J.-C. Poggiale and J. L. Zhou

Copyright (c) 2013 A. V. Kazansky and A. A. Shupikova. This is an open access article distributed under the Creative Commons Attribution License, which permits unrestricted use, distribution, and reproduction in any medium, provided the original work is properly cited.

\begin{abstract}
The aim of this study is the verification of a new velocity-based feature model, called streamlets, proposed recently for objective analysis of the three-dimensional velocity structure of jet streams and eddies in the oceans. Streamlets are continuously imbedded shearing vortex solenoids having two forms: cylindrical (for jets) or toroidal (for eddies, considered as self-closed jets). Both these forms comprise stream coordinates based on streamlines of maximum velocity as an axis and vertical velocity cross-sections defined as an oblique cone with elliptical base. Assimilation of velocity measurements is accomplished by fitting this cone to available data using the well-known Nelder-Mead simplex downhill algorithm for finding the minimum of nonlinear parametric functions. Advantages of the streamlet model are discussed emphasizing its functional integrity. The focus is on velocity data assimilation based on coherency of synoptic scale features as opposed to usual pointwise assimilation methods such as averaging or optimal interpolation. Case studies present synoptic features of a different origin and scale including surface-intensified and subsurface baroclinic examples as well as deep barotropic ones demonstrating universality of the model. The theory of streamlets is also addressed in this paper, since it further sustain the streamlet model.
\end{abstract}

\section{Introduction}

Feature modeling of jet streams and eddies, termed later as synoptic features for short, is a new and very promising kind of an objective analysis of oceanographic fields. In this analysis, the so-called velocity-based feature models intended for objective analysis of velocity fields take on special significance. The reason is that by determining ocean dynamics these fields rule all other fields. However, due to their high spatial and temporal variability, it is hard to reproduce them by means of formal and instable correlations used in traditional objective analysis (for instance, in the standard technique of "optimal interpolation"). We also should take into account that velocity measurements (in comparison to the see surface temperature, for instance) are rare and asynchronous (buoy, by means of drifters observation, Acoustic Doppler Current Profiling, or ADCP) or incomplete (indirect one-component measurements with satellite altimetry). Moreover, most measurements are located in the near-surface layer, and there is a need to extend them deeper. The advantage of feature models (in comparison to point methods of objective analysis of oceanographic fields) is their integral character, which enables us to solve this very important problem efficiently.

Another problem, which has appeared in relation to development of numerical models of oceanic currents, is the challenging problem of data assimilation, known as the initialization problem of numerical forecasting. It consists in extracting the semipermanent (balanced) components of the flow by separating them from high-frequency transients: unbalanced truncation errors or unfiltered nonstationary components can induce unacceptable spurious motions. 
Although numerical models usually include assimilation schemes that utilize coherency (correlation) properties, they require some knowledge about stream function for projecting raw data into the so-called "slow manifold." Of course, such projecting requires nonlinear transformation of data that is necessarily derived from these same data. Because of the nonlinear nature of synoptic features, usual filters and interpolation schemes, tuned to reduction of independent measurement errors, solve this problem badly. For instance, an application of the standard "optimal interpolation" leads to patchiness of the velocity fields and its derivatives. In contrast, feature models due to their functional integrity can be tuned to filter small-scale disturbances, so this technique is promising for initialization and reinitialization of modern eddy-resolving numerical models [1, chapter 15.5]. We should also note that although feature models are simple nonlinear analytical functions, their composite could produce complicated fields. Examples of such fields can be found in [2].

Finally, an important property of the velocity-based feature models is the direct introduction of the so-called stream coordinates, which enable us to improve substantially the representation of the gradients for any oceanographic fields (physical, chemical, and/or biological). Previously, for example, in $[3,4]$, this approach was used only for obtaining average cross-sections of jet streams but there is the need to apply it for all synoptic features, including individual transects of eddies. It is important to note that our definition of stream-coordinates is different from others; it is based on the lines of maximum velocity.

The basic problem with feature modeling is the lack of universality; the velocity-based feature models are not in exclusion since all previously proposed models (e.g., [2, 5]) include empirically selected functions, which must be heuristically assigned for any particular synoptic feature. Unlike these empirical models, the streamlet model offered in the communication [6] is universal. This is because the underlying rational theory of streamlets is based on the newly discovered nonlinear inertial balance, which does not depend on the origin, scale, and even points in time of synoptic features. The theory of streamlets is also addressed here, since the perspective of this paper is to provide observational evidence based on available measurements of several synoptic features to sustain this theory.

\section{The Theory of Streamlets}

2.1. The Streamlet Model. Streamlets consist of an axial line of maximal velocity determining stream coordinates and a cross-section defined as an oblique cone with elliptic base. The theory of streamlets incorporates a priori physical knowledge of rotational and three-dimensional flows as composed of continually nested vortex solenoids with isovelocity (isotach) Lamb surfaces having cylindrical (for jets) or toroidal (for eddies, considered as self-closed jets) forms. An upper part of those synoptic features creeping out the free surface is chopped off and replaced with a vortex sheet (Figure 1). It is important to note that such chopping-off can have place only on the free surface, where a huge jump of

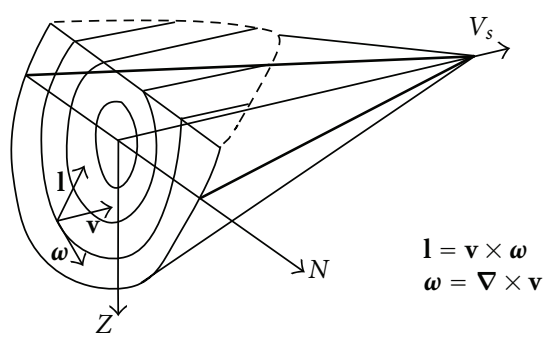

FIgURE 1: Velocity cone of streamlet. The section with maximal velocity contains a triangular profile, while others contain hyperbolic ones. The vectors shown are velocity $(\mathbf{v})$, vorticity $(\omega)$, and Lamb (1) vectors.

density exists, but nowhere else. This "chopping" also means collapsing the Lamb surfaces into thing layers rather than breaking them.

2.2. Preliminaries. Our standpoint in developing the theory of streamlets is that the flows of interest are rotational and three-dimensional, so the appropriate tools for capturing their quantitative behavior are the Lamb vector and surfaces. We intend to explain by that the distinctive properties of synoptic features, namely, their coherency and compactness. For rotational flows to be described properly, one needs to consider a nonzero vorticity vector,

$$
\omega \equiv \nabla \times \mathbf{v},
$$

where $\mathbf{v}$ is the $3 \mathrm{D}$ velocity vector.

Just by the definition (1), three assertions important for studying coherent structures can be made. First, vorticity field is divergent-free (solenoidal),

$$
\nabla \cdot \omega=0 .
$$

Second, the velocity vector is everywhere perpendicular to the vorticity vector (null helicity density),

$$
\mathbf{v} \cdot \omega=\mathbf{v} \cdot \nabla \times \mathbf{v}=0 .
$$

This is because the curl operator acts perpendicular to the source vector. For rotational flows, it can be rigorously proved using the Clebsh transform [7, chapter 2.3.3].

Third, the nonzero vorticity vector allows for introducing the Lamb vector,

$$
\mathbf{l} \equiv \mathbf{v} \times \omega,
$$

which orients the Lamb surfaces formed by orthogonal crossing families of stream lines and vortex lines. The most amusing peculiarity of the Lamb surfaces is the trick of locking up both three-dimensional velocity and vorticity vectors into two-dimensional manifolds. Recall that in order to satisfy the assertion (3) in two-dimensional rotational flows, vorticity is assumed unphysical and one dimensional.

Next, as was proved in [8], the necessary and sufficient conditions of the Lamb surfaces existence are $\mathbf{1} \cdot \nabla \times \mathbf{l}=0$, $\mathbf{l} \neq 0$. However, these conditions look redundant (and thus 
not necessary), since the first condition follows from the second one due to the properties of the curl operator (as in the previous case of helicity density). There is a more simple way to understand this fact. Indeed, the Lamb vector is perpendicular to both velocity and vorticity vectors by definition, and this may only be possible if these later vectors belong to a two-dimensional manifold, that is, a surface. So we came to the following.

Proposition 1. The necessary and sufficient condition of the Lamb surfaces existence is simply the existence of nonzero Lamb vector (4).

And this later property, in turn, is ensured for rotational (i.e., vorticity-driven) flows, which thus may be considered as the flows with Lamb surfaces. According to (2), the existence of Lamb surfaces in any rotational flow indicates the necessity for them to be concentrated in volumes with dense distribution of vorticity. Such compact volumes must be edged by surfaces with zero Lamb vector (i.e., with zero either velocity or vorticity) called critical surfaces (which may degenerate into lines-e.g., the lines of maximal velocity).

Another side of the coin is an important property of the Lamb surfaces in no way to be chaotic [7]. Consequently, such flows may be considered as laminar (specifically, complex lamellar), although not obviously globally connected. Notice that global connectedness is the distinctive attribute of simple laminar flows, while turbulent flows may be described by multiple disconnected pieces [9]. So, every particular synoptic object may be considered as laminar (or coherent).

To proceed further, we need to prove the following.

Proposition 2. The Lamb surfaces are isotachic (iso-velocity). So, the magnitudes of velocity and kinetic energy density can vary only on normal to the Lamb surfaces, $\mathbf{n}$.

Proof. There cannot be a vorticity flux through the Lamb surfaces by definition. Consider a closed contour (loop) composed of two arbitrary streamlines and bridges with null velocity (nominally vortex lines) on a Lamb surface. Applying the Stokes' theorem, the circulation of velocity in this loop is zero. So, velocity is homogeneous at the Lamb surfaces, that is, these surfaces are isotachic. As a result, the magnitudes of velocity, $q=|\mathbf{v}|$, and kinetic energy density (hereafter $K E$ ), $K E=(1 / 2) q^{2}$, can vary only on normal to the Lamb surfaces, n.

2.3. Nonlinear Inertial Balance. We can disclose now the main physical mechanism maintaining our synoptic features in the following.

Proposition 3. The nonlinear inertial balance (NIB) holding for flows with Lamb surfaces annihilates the nonlinear term of the usual momentum (Navier-Stokes) equation:

$$
(\mathbf{v} \cdot \nabla) \mathbf{v} \equiv \nabla K E-\mathbf{l}=0 .
$$

Proof. From Proposition 2 follows a simple expression for the magnitude of vorticity, $\zeta=|\omega|$, as the velocity shear along the normal $\mathbf{n}: \zeta=\partial q / \partial n$. Then, by combining (3) and (4), we obtain the expression for the magnitude of the Lamb vector, $l=q \zeta$, as $l=q \partial q / \partial n$. At the same time, disclosing the gradient of $K E$ yields $(1 / 2) \nabla q^{2}=q \partial q / \partial n$, or in the vector form, $\nabla K E=l \mathbf{n}$. From (4), $\mathbf{l}=\mathbf{l} \mathbf{n}$, so substitution of these into the well-known vector identity gives (5).

The NIB answers the principal question stated in [10] as follows: "there is a mystery as to why jets exist at all-why is there this propensity to concentrate energy and momentum?" The maintenance of jets is explained by NIB as that: the Coriolis acceleration (the Lamb vector) intrinsic to any rotational flow induces the gradient of $K E$, resulting in its concentration in a compact jet cross-section.

2.4. Topology. It is promising now to apply the NIB to infer the topological structure of our synoptic features. As a starting point, let us consider the three-dimensional stationary (steady state) divergence-free analytic flows described by the stationary Euler equation as follow:

$$
(\mathbf{v} \cdot \nabla) \mathbf{v}+\nabla p=0
$$

A virtually complete description of such flows is given by the classification theorem proven by Arnold [11, chapter 2, Theorem 1.2] in 1965 as follow:
Assume that the region $\mathbf{M} \subset \mathbf{R}^{3}$ is bounded by a compact analytic surface, and that the field of velocities is analytic and not everywhere collinear with its curl. Then the region of the flow can be partitioned by an analytic submanifold into a finite number of cells, in each of which the flow is constructed in a standard way. Namely, the cells are of two types: those fibered into tori invariant under the flow and those fibered into surfaces invariant under the flow, diffeomorfic [sic] to the annulus $\mathbf{R} \times S^{1}$ (see Figure 9 in [11]). On each of these tori the flow lines are either all closed or all dense, and on each annulus the flow lines are closed.

We need to make some revisions to this theorem.

First, the two types of flow structures are typified by nested tori on which flow lines are twisted about without being knotted and annular motions in which every fluid particle moves on a circular orbit about a fixed axis of symmetry (but keep in mind that it admits diffeomorphic equivalents to these field lines). The most disturbing aspect of this later flow structure is that it interprets eddies as the "monopoles," which are vortices composed entirely of either positive or negative vorticity [7, chapter 6]. But such models have neither physical meaning (as well as magnetic monopoles) nor mathematical grounds (as inconsistent with the basic property of vorticity to be nondivergent). Thus, these monopole annuli should be disregarded.

Second, there may be another type of flow structures from torroidal and annular, namely, cylindrical ones. Indeed, it is a priori assumption that the region $\mathbf{M} \subset \mathbf{R}^{3}$ is bounded by a compact analytic surface restricting the flow structures by nested tori. However, this provisional assumption may be 


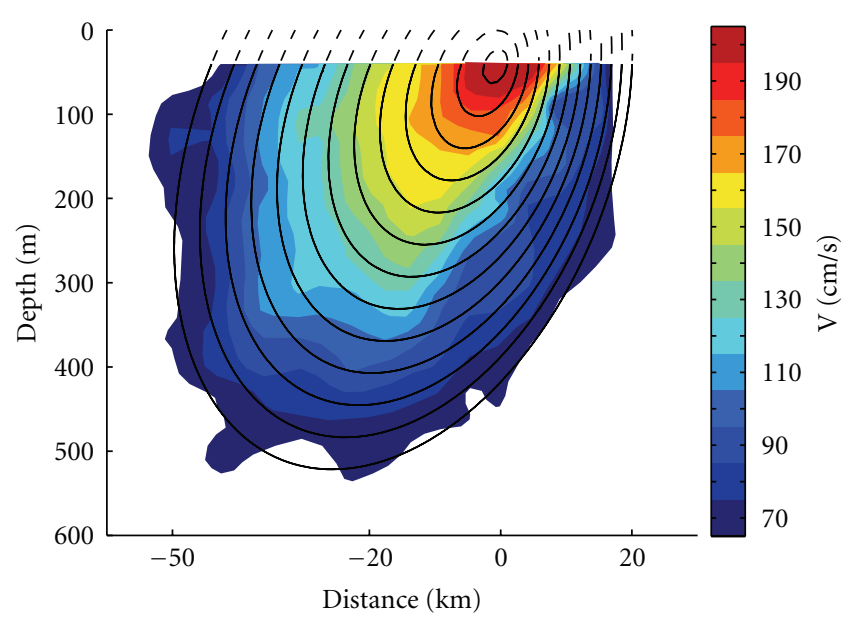

(a)

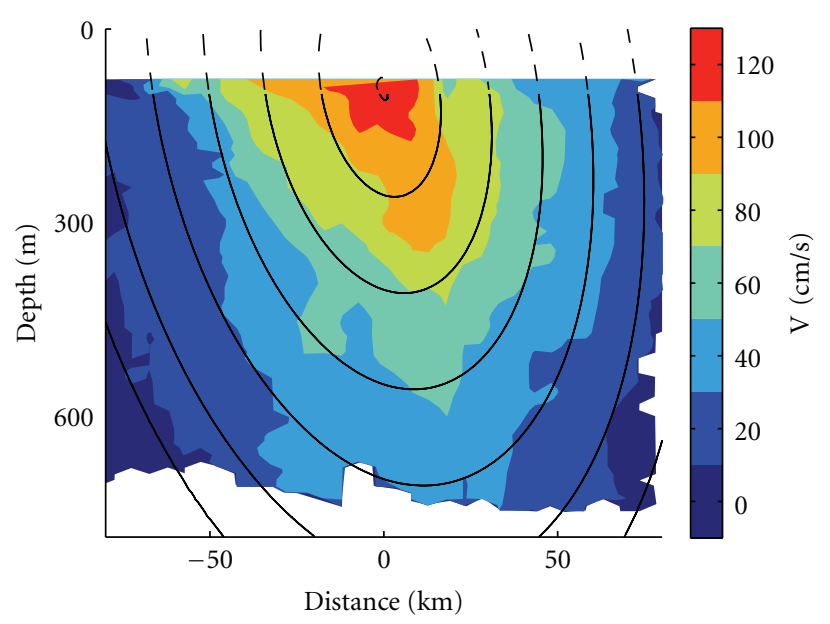

(b)

FIGURE 2: The along-stream velocity section of KE (a) and that of the KE cold core ring (b). Curved lines imposed on the averaged ADCP measurements are modeled by streamlets.

loosening up to allow for semiclosed regions with streamlines to be remotely closed in the direction of flow as virtual cylinders.

Third and final, both the pressure gradient and stationary condition in fact do not play any role in the proof of this theorem [11, chapter 2, section 1.B]. Actually, (6) in the proof was rewritten as $\mathbf{v} \times \omega=\nabla \alpha$, with Bernoulli function $\alpha=p+(1 / 2) q^{2}$. But all that was really used is a level function property of $\alpha$, which arranges the whole structure of corresponding $\alpha$-level (i.e., Lamb) surfaces. As has been already pointed out, the nonlinear inertial balance holding for rotational flows involves the Lamb vector and the kinetic energy density but no other terms, so $\alpha=(1 / 2) q^{2}$. This fact allows us to relax the main assumptions of the Arnold's classification theorem and to use the full form of momentum equation with viscous term, nonuniform density distribution (i.e., $p / \rho$ ), conservative as well as nonconservative forces, and even to drop the assumption of steady state-the NIB is established instantly in any smoothly-deforming continuum. All these terms are involved in their own balances and do not influence the final result: toroidal or cylindrical families of continuously nested Lamb surfaces describe the topological structure of any synoptic feature, independently of its origin, scale, and time. This fact makes the solid ground for the streamlet model, yet this basic model, having vorticity closed in loops in cross-sections perpendicular to all streamlines, may be further generalized to include curved streamlines and vortex lines to become a universal model of coherent structures.

2.5. Geometry. Although topological methods cannot directly provide information about the geometry of synoptic features, with the assistance of NIB we may arrive at some conclusions about their form and velocity-vorticity distribution. Namely, the NIB shapes up the Lamb surfaces cross-sections made of smooth unknotted loops (or ovals); the appearance of inflection points on them leads to disrupting the NIB and, as a result, to splitting synoptic features. This is the main mechanism of rings shedding from jet streams as well as jet separation. As the first approximation, these cross-sections of isolated jets or eddies can be described with ellipses. It is important to note that this mechanism acts in the presence of exterior forces, including buoyancy forces and the planetary Coriolis force. One of the revelations of the planetary Coriolis force is an asymmetry in vorticity distribution, which we reproduce by inclination of the velocity cone, the velocity maximum does not coincide with the center of the elliptic base.

It is much more difficult to find the velocity (as well as $K E$ and vorticity) distribution inside synoptic features, i.e., to get to know how velocity changes with the transition from one Lamb surface to another. All that we know until now is that the velocity must increase toward the critical axis of the velocity maximum due to NIB, while the rate of this increase is uncertain. Since the velocity distribution inside jets and eddies must depend on how the flows were established, we need to consider the mechanisms of the jets and eddies formation.

The main difficulty with studying the prehistory of the jet streams and eddies in the ocean is that these features are forced by turbulence and thus have "a multiscale memory." Nowadays, it is a widespread convincement that jet streams and eddies are not the elements of oceanic turbulence but represent the so-called mean currents existing on turbulent background-see, for example, [12]. Unfortunately, this hypothesis is incompatible with the fact that there is no such thing as the large scale forcing-all known forcing mechanisms (wind stress, convection, baroclinic production, and topography drag) produce small-scale turbulence, and so we need to explain how the large-scale jets and eddies may originate from a small-scale forcing (i.e., to clarify mechanisms of an upscale energy transfer).

Although the NIB does not depend on scale, it can indirectly help to understand the physical mechanism detaining 


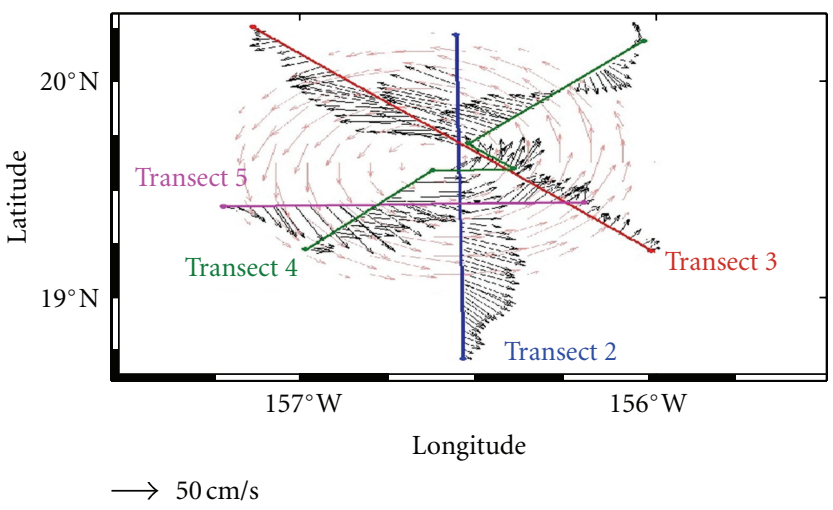

(a)

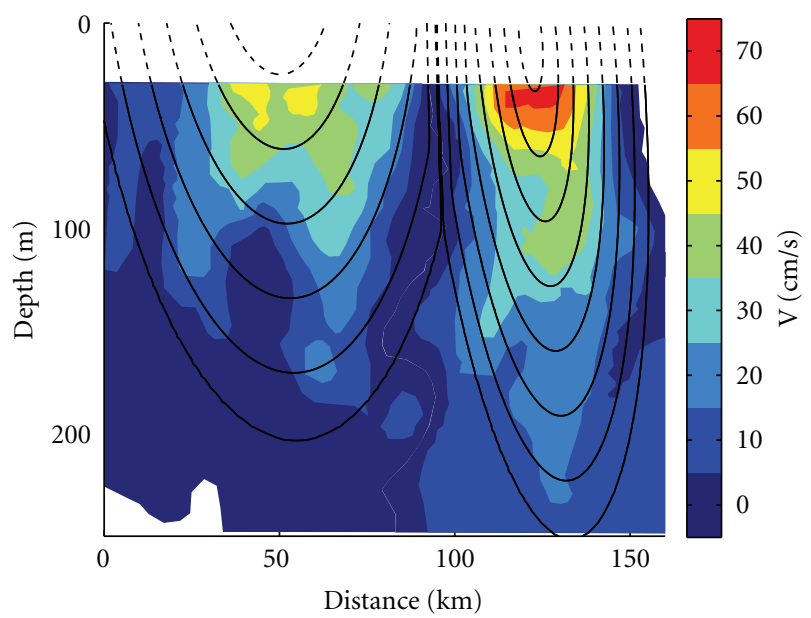

(c)

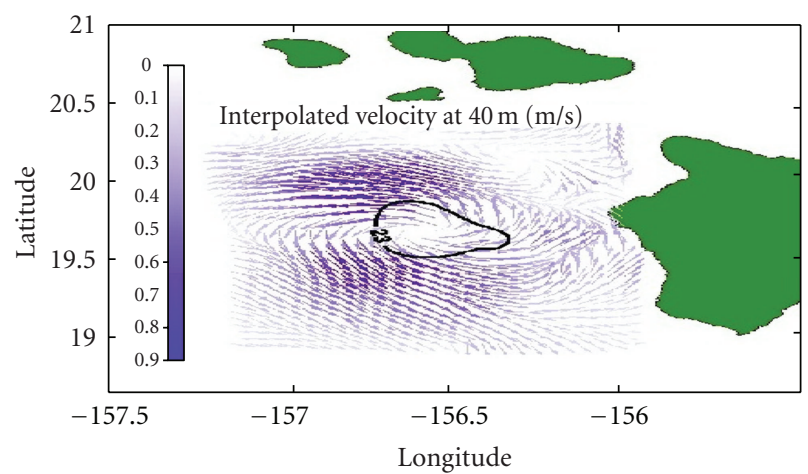

(b)
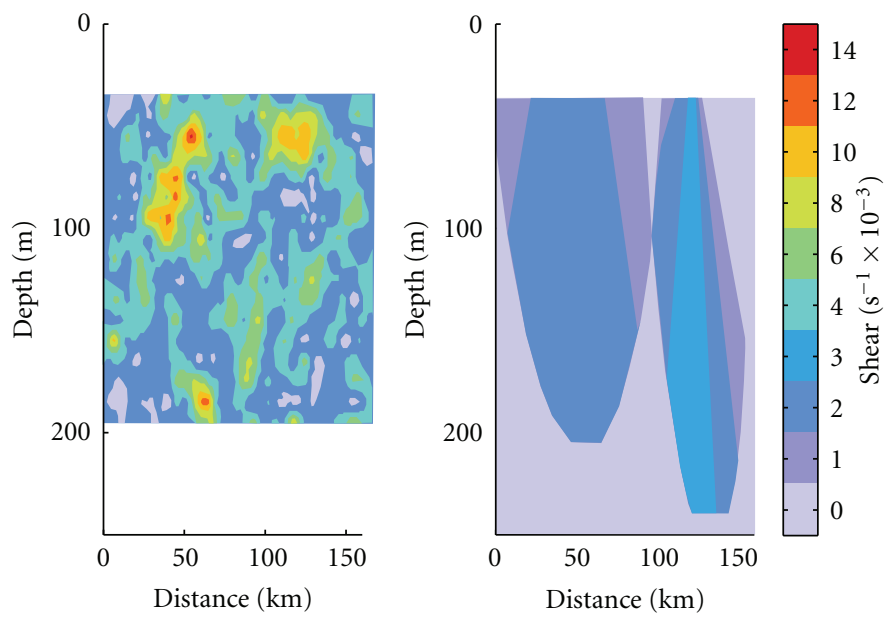

(d)

Figure 3: Hawaiian eddy Noah. (a), (b) compares horizontal sections of this eddy velocity field, obtained with the streamlets ((a), pink vectors) and from the pure interpolation (b); (c) shows the vertical velocity cross-section, and (d) pictures the vertical velocity shear derived by optimal interpolation (d) left and using streamlets (d) right.

energy in large-scale synoptic features. Let us consider a velocity profile $q(L)$, where $L$ is taken along the gradient of $K E$ beginning from outer margin with zero velocity. Note that the NIB assures a monotonic velocity raise between critical points (from zero to maximum), so that vorticity does not change sign on this profile. Defining an average vorticity on the interval $(0, L)$ as $\left\langle\zeta_{L}\right\rangle=q(L) / L$ gives an estimate of the rate of $K E(L)=(1 / 2) q(L)^{2}$ to the average enstrophy, $E n(L)=(1 / 2)\left\langle\zeta_{L}\right\rangle^{2}$ as

$$
\frac{K E(L)}{E n(L)}=L^{2}
$$

The estimate (7) coincides with integral estimations based on the Fourier analysis [7, chapter 5.8] but departs from them by a differential representation of locations with different velocity distribution.

Now we can clarify the physical mechanism detaining energy in growing synoptic features. According to this estimate, the larger feature, the lesser enstrophy it can hold. Consequently, for $K E$ to transfer upscale (or penetrate deeper), it is necessary only to get rid of redundant vorticity, which is easy to achieve by stripping off vorticity in the form of even smaller filaments, while the most of $K E$ retained in a large-scale synoptic feature. In contrast, to go downscale, an additional (secondary) vorticity must be generated. Therefore, the estimate (7) signifies the trap for KE in the innermost parts of coherent structures, where the ratio $K E(L) / E n(L)$ is large and there is simply not enough vorticity for $K E$ to escape. The downscale energy transfer is possible due to different instabilities, but oceanic synoptic features are too well stabilized by stratification and planetary rotation to became turbulent. So, their fate is to evolve much more slowly than the turbulence by losing energy through edges where the low $K E / E n$ makes such slow decay possible.

So, the NIB implies the formation of synoptic features through the upscale energy transfer driven by turbulent diffusion (eddy forcing) assisting to physical explanation of the velocity cone of streamlets. Because any diffusion is always directed at elimination of gradients, one can expect smoothing down the vorticity distribution during the formation stage-it is the physical explanation for the velocity cone of streamlets [13]. 


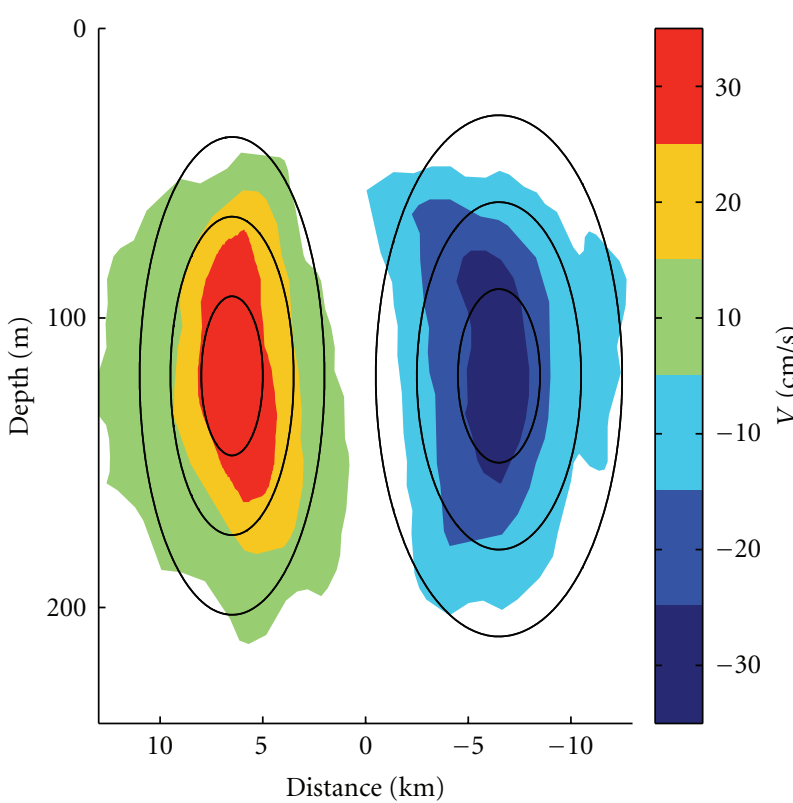

(a)

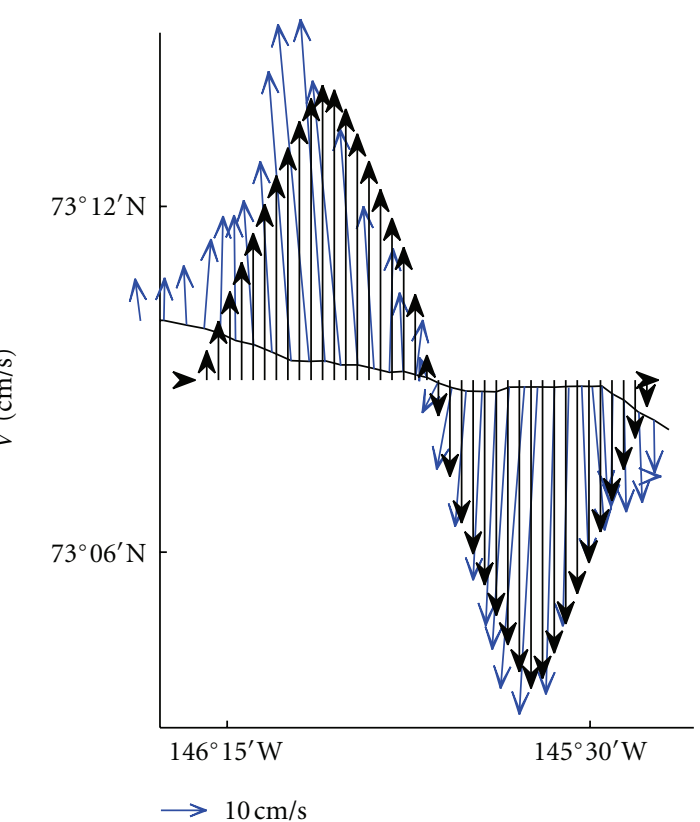

(b)

Figure 4: Halocline eddy example from B92 IOEB ADCP data showing (a) contours of current velocities relative to the background by depth and radius from the eddy center, and (b) current vectors at depth of eddy center (104 m) superimposed on IOEB drift (in blue) with superimposed streamlet-modeled vectors (in black).

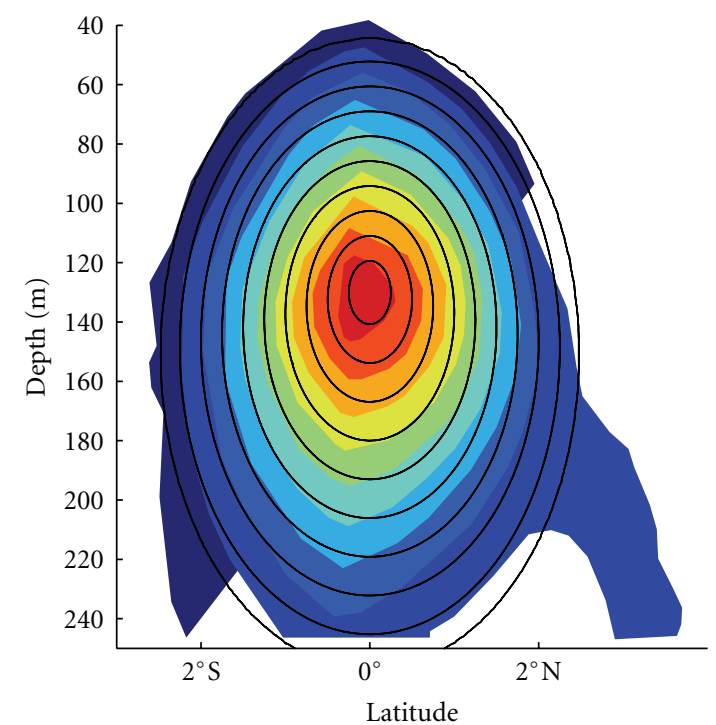

(a)

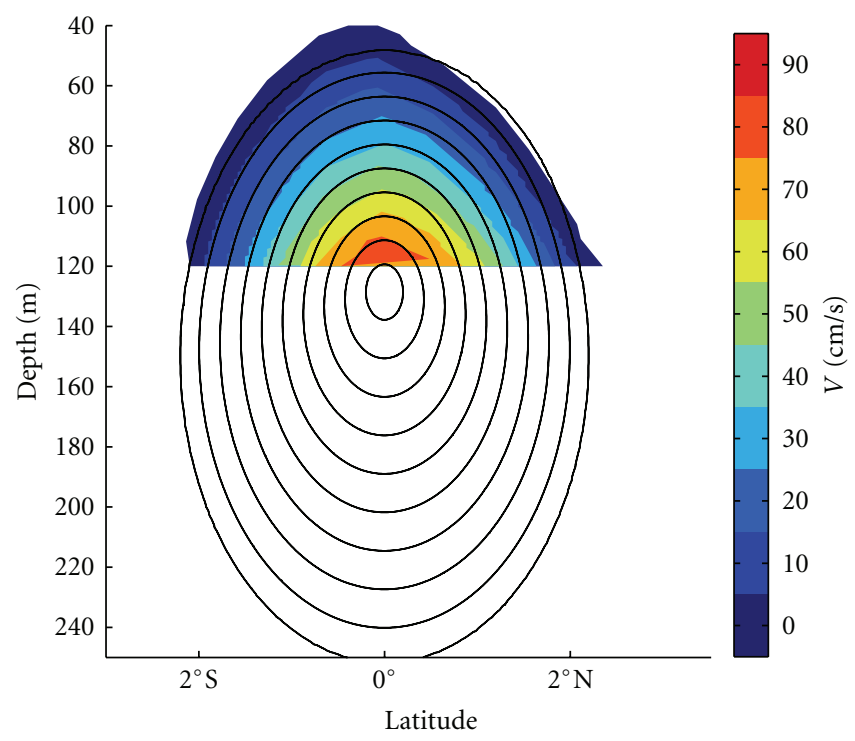

(b)

FIgURE 5: (a) The vertical velocity cross-section of EUC obtained using ADCP plus PCM data. (b) The same using only ADCP data.

\section{Observational Evidences}

This section presents observational evidences of the velocity structure of synoptic features with additional assignments to illustrate different abilities of streamlets in solving the previously mentioned problems of objective analysis of velocity data. All case studies were carried out by fitting the velocity cone of streamlets with the help of the well-known NelderMead simplex downhill algorithm for finding the minimum of objective functions with nonlinear parameters: eight for the vertical or horizontal cross-section, presuming an elliptic form of the later.

3.1. Surface-Intensified Synoptic Features. A dual case study of the Kuroshio Extension, KE, and the KE-"pinched" cold core ring illustrates the similarity of both velocity field structures, which are baroclinic (Figure 2). The data used were from 


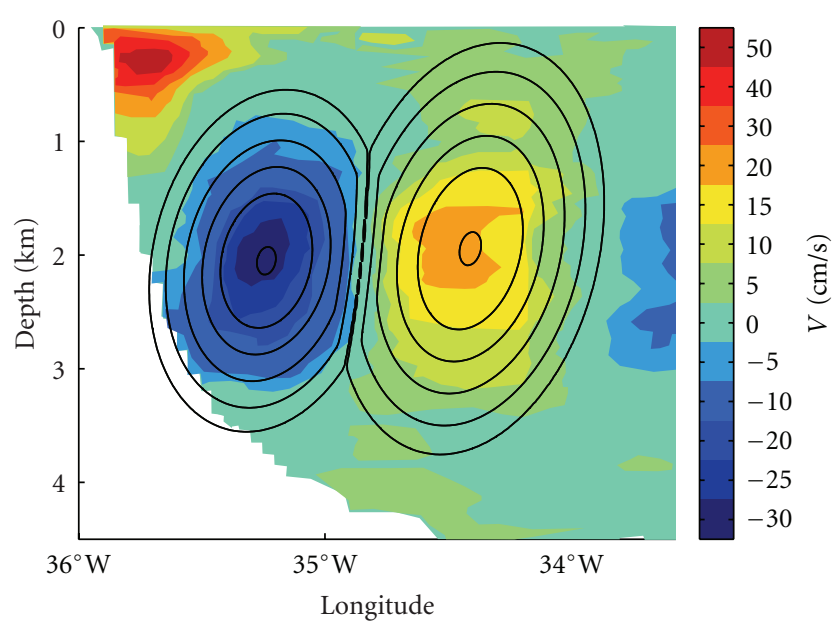

FIGURE 6: Atlantic deep western boundary current eddy observed with lowered ADCP measurements (in color) and streamlet-fitted black curves.

averaged lowered ADCP measurements: for the KE from [14], and for the KE cold core ring from [15].

The second example is a mesoscale eddy feature of a quite different origin: the wind-stress generated cyclone Noah; quasisynoptic observations of its horizontal and vertical velocity structure were conducted in the lee of Hawaii [16]. This example demonstrates abilities of the streamlet model to represent the velocity structure with individual (not averaged) measurements and also shows its capability to evaluate the velocity derivatives.

The top panel of Figure 3 shows the streamlet-based reconstruction of the horizontal velocity section of this eddy in Figure 3(a), which has been preconditioned to be elliptic, compared with the interpolated velocity field in Figure 3(b), as in [16]. The curvature correction for this reconstruction accounts for changes in widths of the triangular maximum velocity cross-stream profiles; it assumes that the vorticity (vertical on this profile) conserves along horizontally curved streamlines, so the corresponding velocity profile obeys the rule

$$
-\frac{\partial V}{\partial n}+\frac{V}{r}=\omega,
$$

where $\omega$ means the zero-curvature vorticity. This gives curvature correction for this profile as

$$
V(n)=\frac{\left[V_{\max }+\omega_{L, R} R\left(\rho^{2}-1\right) / 2\right]}{\rho}, \quad \rho=\frac{r}{R},
$$

where $R$ is radius of curvature with respect to the maximum velocity, $r=R-n$ is the local radius of curvature, ans $\omega_{L, R}$ is a parameter meaning the zero-curvature vorticity to the left or to the right of the stream axis (velocity maximum). Let us compare this result with a qualitative description in [17, chapter 18.1.2].

Figure 3(c) presents interpolated velocity measurements from Transect 3 fitted with the streamlet model. The vertical structure of the vertical shear of velocity (horizontal vorticity) is presented in Figure 3(d) right. Great sensitivity of velocity derivatives to small-scale disturbances produces patchiness in optimal interpolation results as in Figure 3(d) left. Functional integrity of the streamlet model describing both velocity and vorticity structures grants efficiency to streamletassimilation scheme.

As to the averaging, notice that sections are different because shallow water conditions deform the eddy velocity structure. Not only averaging cannot be applied in this case, but also an additional procedure of volume rendering is required to continuously connect different vertical crosssection to be a valid $3 \mathrm{D}$ model. Developing such a procedure is the subject of ongoing work.

3.2. Subsurface Examples. A halocline Arctic eddy from $B 92$ IOEB ADCP data [18] exemplifies subsurface "meddy"-type eddies (Figure 4). This particular eddy displays the triangular velocity profile and toroidal velocity structure characteristic to the streamlet model. A peculiar aspect associated with this high latitude eddy is negligible $\beta$-effect, which is often advocated as being vital for the existence of synoptic features.

The Equatorial undercurrent, EUC, gives a distinctive example of the velocity cone of streamlets. The data presented in Figure 5(a) were made from synthetic observations (ADCP plus PCM) [19]. Figure 5(b) demonstrates the model ability to extend data from near-surface layer (down to $120 \mathrm{~m}$ deeper). The peculiarity of this example is that the geostrophic balance does not hold for EUC, although the thermal wind balance does, yet to compensate the horizontal shear of velocity from the usually implied vertical one. This fact clearly reveals that contrary to the widespread opinion, the geostrophic balance does not maintain synoptic features.

3.3. Deep Barotropic Eddies. Interpolated lowered ADCP measurements of an Atlantic deep western boundary current eddy [5] show toroidal velocity structure with the noticeable vertical shear of velocity. Superimposed are the streamlet model curves (Figure 6).

The Labrador eddy F, observed from a mooring (Figure 7), displays the same toroidal velocity structure [20]. In addition to its deep extension, it has very weak stratification implying barotropic conditions from top to bottom.

These two deep eddies extending well below pycnocline are mostly barotropic at least in their lower parts and yet noticeably vertically sheared. This contradicts to the wellknown Taylor-Proudman theorem prohibiting the change of the velocity with depth for barotropic features but further sustains the streamlet model allowing such velocity shear.

\section{Conclusion}

This paper provides observational evidence to support the streamlet velocity-based feature model proposed recently for objective analysis of the velocity field structure of jet streams and eddies. The underlying theory revealed nonlinear inertial balance as the main mechanism maintaining synoptic features. This balance between the Lamb vector (physically 


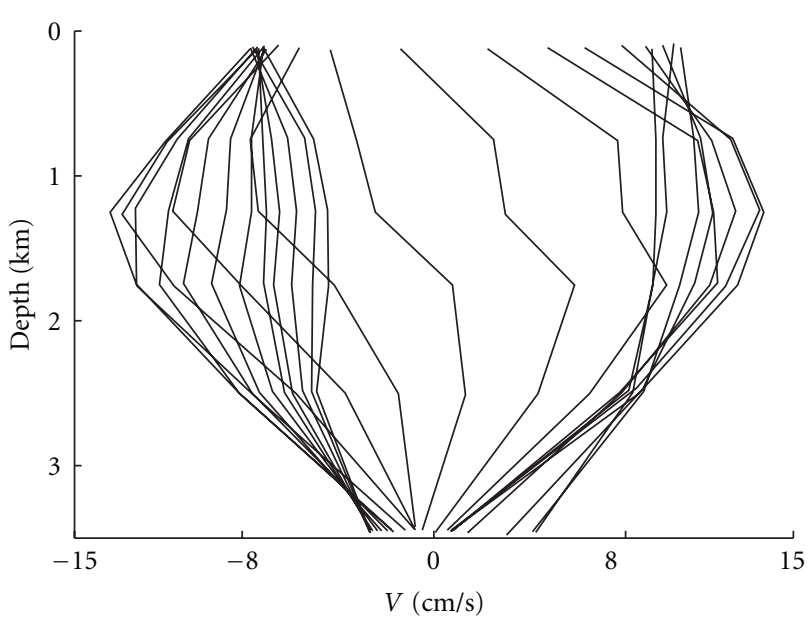

(a)

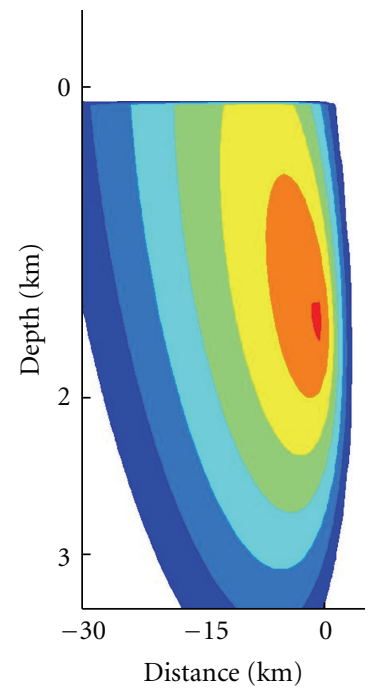

(b)

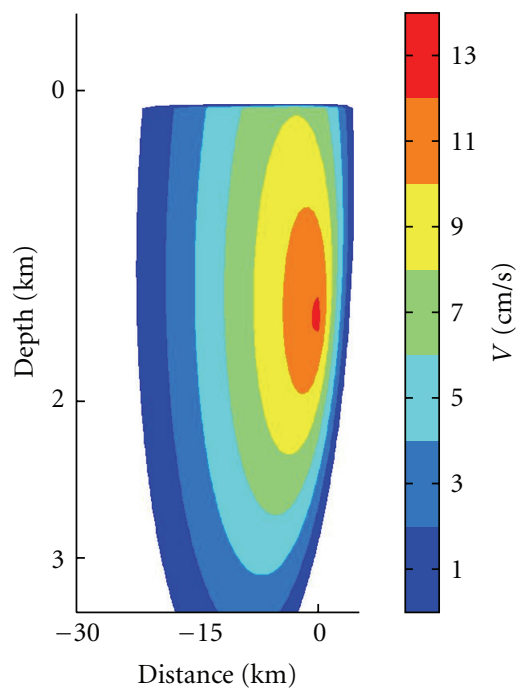

(c)

FIGURE 7: The Labrador eddy F. The original mooring data are on left (a); the reconstructed velocity sections are at right ((b) and (c)).

the Coriolis acceleration of a velocity field under the effect of its own rotation) and the gradient of kinetic energy density, $\mathrm{KE}$, representing the inertial force was overlooked in previous theories. An important feature of NIB is its independence of origin, scale, and time-it is universal balance of flows with Lamb surfaces. The cylindrical and toroidal forms of coherent structures were confirmed using topological methods combined with NIB.

To demonstrate universality of the model, we present synoptic features of different origin, and scales including surface-intensified and subsurface baroclinic examples as well as deep barotropic ones. The universality of the streamlet model is explained by the fact that the velocity field structure of synoptic features is wholly determined by their internal nonlinear dynamics, which is common for all coherent currents, namely, because these features are maintained by the newly discovered NIB, which does not depend on their nature.

In passing, we display specific abilities of the streamlet model to estimate velocity derivatives, to extend them deeper from the free surface layers, and to fit barotropic flows, which are prohibited by the Taylor-Proudman theorem. This puts a baffling question about the validity of this theorem. Although beyond the scope of this study, the theory underlying the streamlet model suggests a probable mechanism for keeping up such vertical shear in barotropic flows by nonlinear terms in the equations of motion omitted in the inference of the Taylor-Proudman theorem.

\section{Acknowledgment}

The Russian Foundation for Basic Research (Project no. 11-0100590-a) supported this work.

\section{References}

[1] R. H. Stewart, Introduction to Physical Oceanography, Department of Oceanography, A\&M University, Collage Station, Tex, USA, 2004.

[2] A. Gangopadhyay and A. R. Robinson, "Feature-oriented regional modeling of oceanic fronts," Dynamics of Atmospheres and Oceans, vol. 36, no. 1-3, pp. 201-232, 2002.

[3] D. Halkin and T. Rossby, "The structure and transport of the Gulf Stream at $73^{\circ}$ W,' Journal of Physical Oceanography, vol. 15, pp. 1439-1452, 1985.

[4] C. S. Meinen, "Structure of the North Atlantic current in stream-coordinates and the circulation in the Newfoundland basin," Deep-Sea Research Part I, vol. 48, no. 7, pp. 1553-1580, 2001.

[5] M. Dengler, F. A. Schott, C. Eden, P. Brandt, J. Fischer, and R. J. Zantopp, "Break-up of the Atlantic deep western boundary current into eddies at $8^{\circ} \mathrm{S}$," Nature, vol. 432, no. 7020, pp. 10181020,2004

[6] A. V. Kazansky and A. A. Shupikova, "On the velocity field structure of jet streams and eddies in the ocean," Doklady Earth Sciences, vol. 431, no. 2, pp. 528-532, 2010.

[7] J. Z. Wu, H. Y. Ma, and M. D. Zhou, Vorticity and Vortex Dynamics, Springer, Berlin, Germany, 2006.

[8] C. A. Truesdell, The Kinematics of Vorticity, Indiana University Press, Bloomington, Ind, USA, 1954.

[9] R. M. Kiehn, "Topological defects, coherent structures and turbulence in terms of Cartan's theory of differential topology," in Developments in Theoretical and Applied Mathematics (Proceedings of SECTAM XVI Conference), B. N. Antar, R. Engels, A. A. Prinaris, and T. H. Moulden, Eds., vol. 3, p. IV.2, University of Tennessee Space Institute, Tullahoma, Tenn, USA, 1992.

[10] M. P. Baldwin, P. B. Rhines, H. P. Huang, and M. E. McIntyre, "The jet-stream conundrum," Science, vol. 315, no. 5811, pp. 467468, 2007. 
[11] V. I. Arnold and B. A. Khesin, Topological Methods in Hydrodynamics, vol. 125 of Applied Mathematical Science, Springer, New York, NY, USA, 1998.

[12] W. D. Smyth and J. N. Moum, "Three-dimensional turbulence," in Encyclopedia of Ocean Sciences, J. Steele, S. Thorpe, and K. Turekian, Eds., vol. 6, pp. 2947-2955, Academic Press, San Diego, Calif, USA, 2001.

[13] A. V. Kazansky, "The theory of streamlets: Nonlinear inertial balance and three-way energy transfer," in Eddies and Hurricanes: Formation, Triggers and Impact, A. Tarasov and M. Demidov, Eds., pp. 19-36, Nova Science Publishers, Hauppauge, NY, USA, 2012.

[14] B. Qiu, S. Chen, and P. Hacker, "Effect of mesoscale eddies on subtropical mode water variability from the Kuroshio Extension System Study (KESS)," Journal of Physical Oceanography, vol. 37, no. 4, pp. 982-1000, 2007.

[15] A. D. Greene, K. A. Donohue, and D. R. Watts, "Vorticity and potential vorticity structure of Kuroshio Extension cold core ring," in Proceedings of the 13th Ocean Sciences Meeting, Honolulu, Hawaii, USA, February 2006, abstract no. OS45G06.

[16] V. S. Kuwahara, F. Nencioli, T. D. Dickey, Y. M. Rii, and R. R. Bidigare, "Physical dynamics and biological implications of Cyclone Noah in the lee of Hawai'i during E-Flux I," Deep-Sea Research Part II, vol. 55, no. 10-13, pp. 1231-1251, 2008.

[17] B. Cushman-Roisin and J.-M. Beckers, Introduction to Geophysical Fluid Dynamics: Physical and Numerical Aspects, Academic Press, 2nd edition, 2011

[18] R. A. Krishfield, A. J. Plueddemann, and S. Honjo, "Eddys in the Arctic Ocean from IOEB ADCP data," Tech. Rep. WHOI-200209, Woods Hall Oceanographic Institution, Woods Hall, Mass, USA, 2002

[19] E. S. Johnson and D. S. Luther, "Mean zonal momentum balance in the upper and central equatorial Pacific Ocean," Journal of Geophysical Research, vol. 99, no. 4, pp. 7689-7705, 1994.

[20] J. M. Lilly and P. B. Rhines, "Coherent eddies in the Labrador Sea observed from a mooring," Journal of Physical Oceanography, vol. 32, no. 2, pp. 585-598, 2002. 

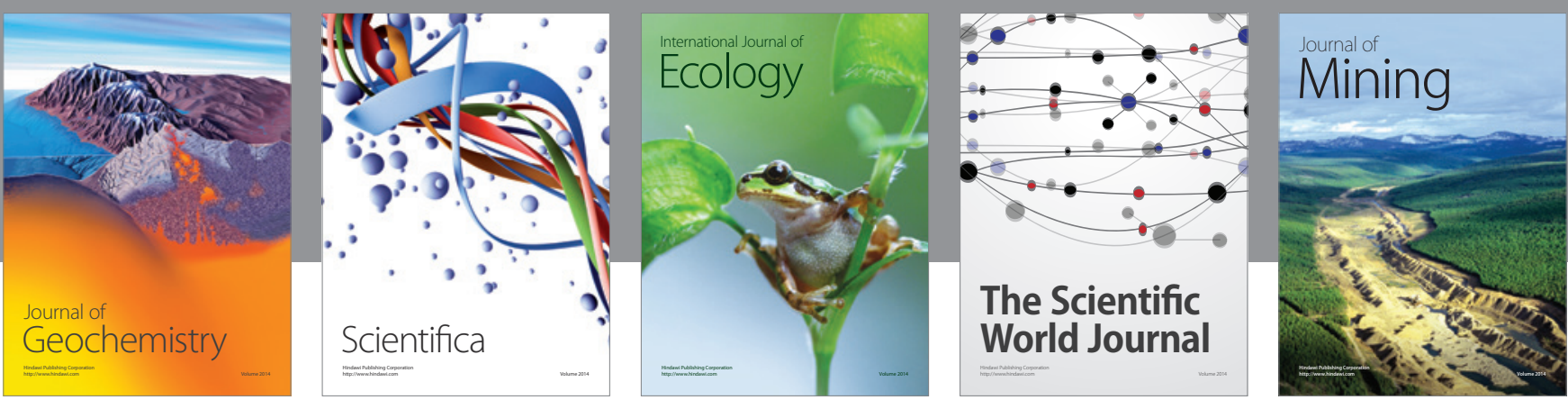

The Scientific World Journal
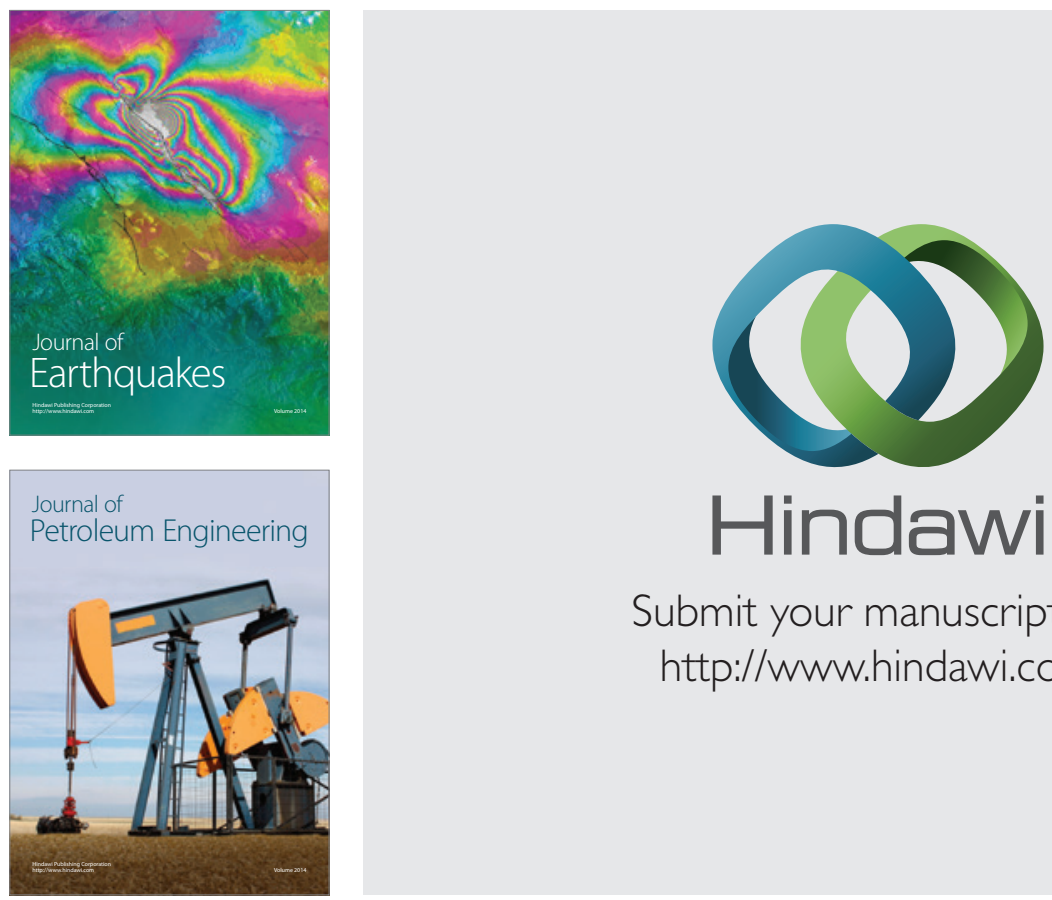

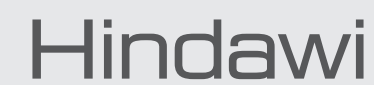

Submit your manuscripts at

http://www.hindawi.com
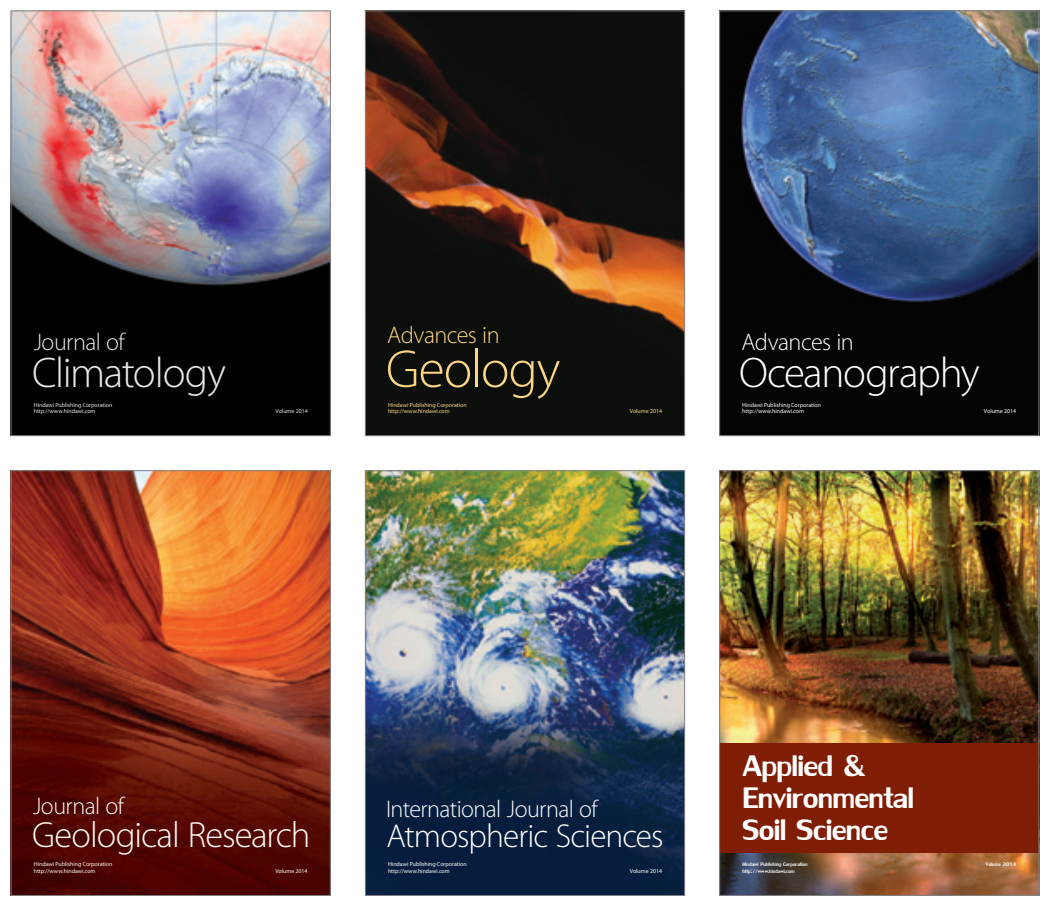
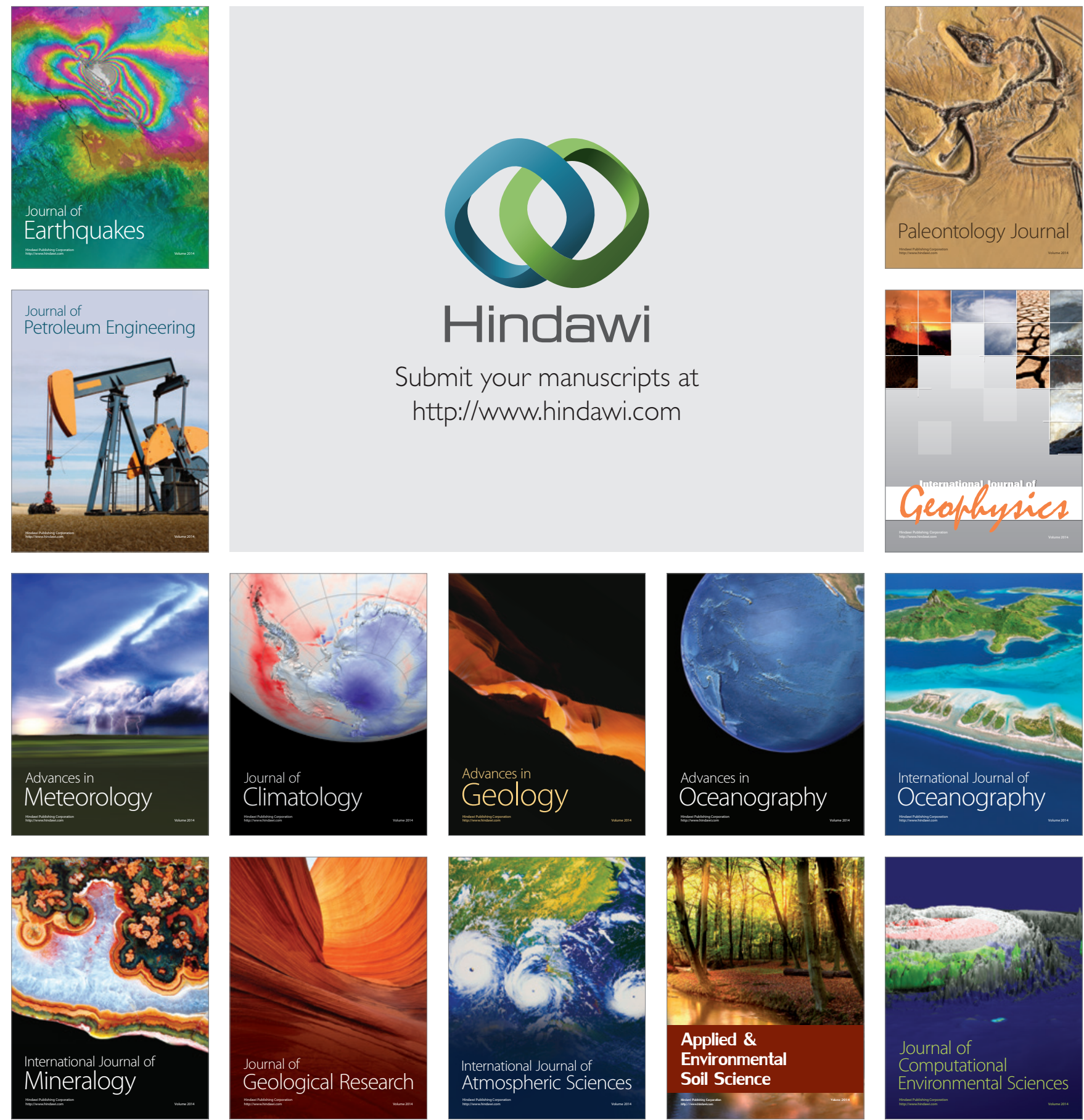